\title{
Three Dimensional Analysis of the Blood Flow Regime within Abdominal Aortic Aneurysm
}

\author{
M. A. Azam and S. A. A. Salam, Member, IACSIT
}

\begin{abstract}
Abdominal aortic aneurysm (AAA) is a degenerative disease, which is defined as the abnormal ballooning of the abdominal aorta (AA) wall usually caused by atherosclerosis. In the present work, finite-volume method for the numerical prediction of non-Newtonian blood flow patterns in AA is performed in the 3D model of AA with aneurysm along with its peripheral branches for systolic and diastolic cardiac phase. Grid independence was tested on three successively refined meshes. It is observed that the abrupt expansion induced by AAA results in an immensely disturbed regime, whereas aneurismal sac is characterized with a multiple vortices and reverse flow in both phases. However, in diastolic phase vortices and reverse flow is more random, and frequent in contrast to systolic phases but with lower kinetic energy. Immense pressure buildup between the renal and iliac bifurcation of AA is observed, that results in a velocity drop across aneurismal sac and unsteady flow at the iliac bifurcation which induces additional stress across the aneurysm. It believes that the pressure gradient is highly responsible for velocity drop, formation of emboli, and continued dilation of aneurismal sac that may result in the obstruction of a blood vessel leading towards rupture of the aneurysm.
\end{abstract}

Index Terms-Abdominal aortic aneurysm, Bio-fluid,CFD simulations, Laminar flow, Non-newtonian blood.

\section{INTRODUCTION}

Abdominal aorta (AA) is the continuation of the thoracic aorta that initiates at the aortic hiatus of the diaphragm and runs down to the body of the fourth lumber vertebra, where the aorta sections in two to form the common iliac arteries [1] Abnormal ballooning of the AA is termed as abdominal aortic aneurysm (AAA), which is usually caused by atherosclerosis [2]. AAA occurs in the infra-renal segment of the $\mathrm{AA}$, between the renal arteries and the iliac bifurcation. Identifying the risk factors associated with the ballooning of AAA leading to its rupture has become pivotal for obtaining a thorough understanding of pathogenesis and evolution of AAA. Still, number of causative factors has been identified among them blood flow hemodynamic and wall shear stress (WSS) distributions are considered the most important factors leading to the abnormal growth, enfeeblement of aorta wall, and rupture of the AAA.

Advancement in the CFD and medical imaging provide an unprecedented opportunity to constructa computational

Manuscript received July 22, 2011; revised October 26, 2011. This work was technically supported by Computational Thermo-fluid laboratory, Department of Mechanical Engineering, NED University of Engineering \& technology, Pakistan.

M. A. Azam is with the Medical Engineer from NED University (e-mail: amerazam87@gmail.com).

S. A. A. Salam is affiliated with Dow University of Health \& Science and Indus Institute of Higher education as Visiting lecturer (e-mail: anzarsalam@gmail.com). domain for the examination of blood flow through the highly convoluted cardiovascular system [3]. In past various CFD analyses have been done for the study of pressure, shear stresses on the aortic wall and predictions of the blood flow regime through AA, but most of them treated blood to be Newtonian fluid. Newtonian and non-Newtonian behaviors of blood are studied by Siebert and Fedor [4]. They infer decreased blood flow velocity is the causative factor behind the transformation of blood behavior from Newtonian to non-Newtonian. Recent investigation includes D. Lee and J. Y. Chen [5], who produced pulsatile flow field through three-dimensional rigid model of the AA, including peripheral branches for both resting and mild exercise conditions, validate that exercise is favorableto health by proving an exercise reduces oscillating wall shear stress and produces a cleaner flow field. D. Lee and J.Y. Chen [6] also examined steady flow field andWSS distribution for the reverse flow regions by applying Finite volume method (FVM). Taylor et al. [7] demonstrated recirculation zone along the posterior wall of the infra-renal aorta by utilizing Finite Element method (FEM) to study blood flow through a rigid model of the AA under resting and exercise conditions.

Several investigators have studied the causative factors of the AAA by utilizing CFD tools for the analysis of blood flow through the AAA. Finol et al. [8] explains the significance of considering time-dependent indicator function of hemodynamic disturbance, which includes wall pressure, wall shear stress and WSS gradient in a two-aneurysm, axisymmetric, rigid wall model by employing spectral element method. Direct relationship of blood pressure with the ballooning of the AAA is demonstrated by Hassani et al. [9] by modeling an electrical system of the cardiovascular system as well as CFD modeling of abdominal aortic aneurysm.

Poullis et al. [10] investigates the importance of aortic curvature $(\mathrm{AC})$ as a potential risk factor by developing a mathematical model. Poullis et al. [10] suggest that AC to be potentially influencing risk factor in comparison to aortic diameter, blood pressure, cardiac output, beta-blocker use and patient size associated with the ballooning of an aneurysm. Khanafer et al. [11], suggests additional stresses across the aneurysm sac to be responsible for wall dilation, results in greater turbulence which in turns initiate a self-perpetuating mechanism for aneurysm growth and rupture by numerical analysis of pulsatile, turbulent, non-Newtonian flow through the axisymmetric aneurysm at rest and during exercise. Khanafer et al. [11] observed small recirculating vortex generated at the proximal end of AAA while a large recirculating vortex noticed in peak diastolic phase, under both rest and exercise.

Evaluation criteria for the risk factors associated with the ballooning of the aneurysm to its rupture is still not full 

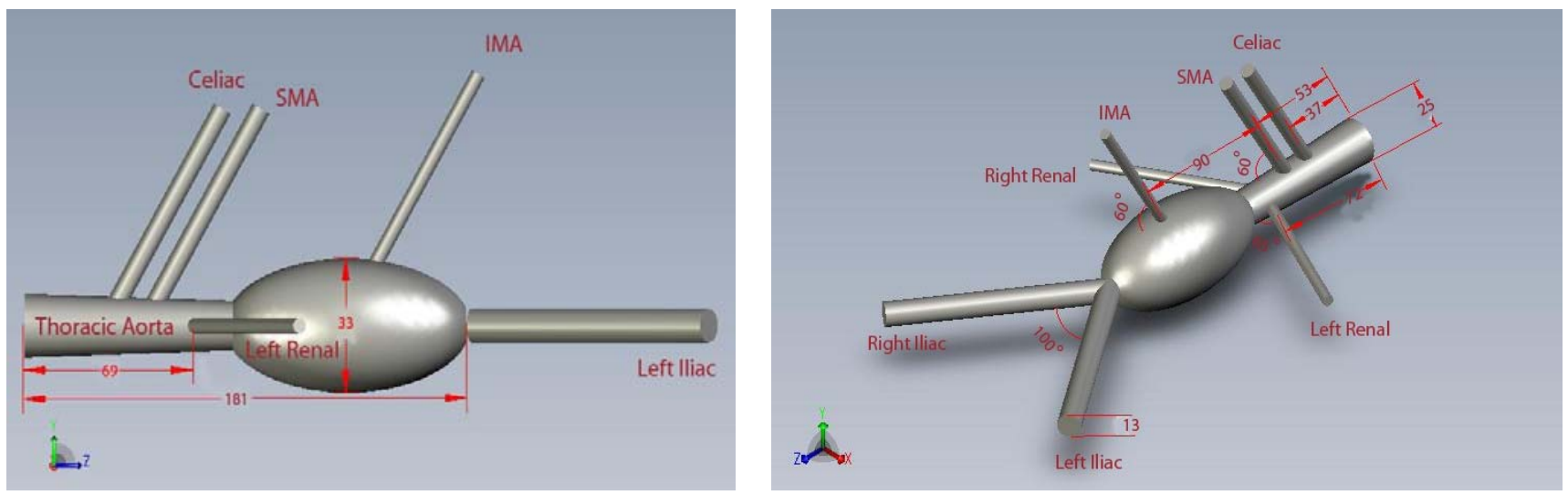

Fig. 1. Geometry of abdominal aortic aneurysm. (IMA= Inferior Mesenteric Artery, SMA = Superior Mesenteric Artery)

understood. In this work, FVM is employed for the numerical prediction of flow characteristics and comparison of blood behavior in a three-dimensional domain of Abdominal Aorta with aneurysm together with its peripheral branches for systolic and diastolic cardiac phase. Blood is assumed to be non-Newtonian, blood flow to be laminar and flow analysis is done by applying the laminar model, using 2 nd order discretization in a space and steady state is assumed.

\section{PROCEDURE FOR PAPER SUBMISSION}

\section{A. Flow model}

Gambit v2.3 is used for the development of a simplified three dimensional model of an abdominal aorta with aneurysm using the approximation from the published values (Table I), along with its peripheral branches like the thoracic aorta, celiac trunk, superior mesenteric, left renal, right renal, inferior mesenteric, left Iliac and right Iliac arteries as shown in Fig.1.

\section{B. Governing equations and boundary conditions}

In this study, blood is assumed to be homogeneous, incompressible, and non-Newtonian fluid; the vessel wall to be rigid, and no slip condition are applied. Laminar model is implemented to study the hemodynamic indicators considering a steady flow. Identical boundary conditions for both diastolic and systolic phases of the blood circulation are applied. Numerical analysis is performed by applying the law of conservation of mass and momentum. Commercial CFD code Fluent v6.3.26 is used to solve governing equations in a computational model of AAA.

Equation 1 depicts the general form of law of mass conservation, whereas law of conservation of momentum is expressed in Equation 2.

$$
\begin{gathered}
\partial \rho / \partial t+\nabla \cdot(\rho \vec{v})=0 \\
\frac{\partial}{\partial t}(\rho \vec{v})+\nabla \cdot(\rho \vec{v} \vec{v})=-\nabla p+\nabla \cdot(\overline{\bar{\tau}})+\rho \vec{g}+\vec{F}
\end{gathered}
$$

where $\rho$ is density of fluid, $t$ is the time, $\vec{v}$ represents the velocity vector, $p$ is the pressure, whereas $\rho \vec{g} \& \vec{F}$ are the gravitational body forces and external body forces respectively. Shear tensor is represented by $\overline{\bar{\tau}}$ and $\mu$ is the viscosity of fluid depicted in equation 3 .

$$
\overline{\bar{\tau}}=\mu\left(\nabla \vec{v}+\nabla \vec{v}^{T}\right)
$$

In case of Newtonian fluid, $\mu$ is considered constant having no effect of shear rate; Non Newtonian fluids have viscosity, which cannot be defined using constant value because of non-linear relationship between shear rate and shear stress. Blood is non-Newtonian [12], [13] because of red blood cells. Investigation shows that at shear stress $<10 \mathrm{~s}^{-1}$ non-Newtonian behavior of blood becomes dominant.

TABLE I: DIMENSIONS OF COMPUTATIONAL DOMAIN

\begin{tabular}{|l|l|l|}
\hline Arteries & Diameter $(\mathrm{mm})$ & Branching Angle \\
\hline Thoracic Trunk & 25 & \\
\hline Celiac Trunk & 8 & $60^{\circ}$ \\
\hline Superior Mesenteric & 8 & $60^{\circ}$ \\
\hline Left Renal & 6 & $65^{\circ}$ \\
\hline Right Renal & 6 & $60^{\circ}$ \\
\hline Inferior Mesenteric & 6 & $60^{\circ}$ \\
\hline Left Iliac & 13 & $50^{\circ}$ \\
\hline Right Iliac & 13 & $50^{\circ}$ \\
\hline
\end{tabular}

TABLE II: NON-NEWTONIAN POWER LAW PARAMETERS

\begin{tabular}{|l|cl|}
\hline \multicolumn{1}{|c|}{ Parameters } & \multicolumn{3}{|c|}{ Description of Parameters } \\
\hline Viscosity Lower limit & $\eta_{\min }$ & $0.00125 \mathrm{~kg} / \mathrm{ms}$ \\
\hline Viscosity Higher limit & $\eta_{\max }$ & $0.00300 \mathrm{~kg} / \mathrm{ms}$ \\
\hline Power law Index & $n$ & 0.48510 \\
\hline Consistency Index & $k$ & $0.20730 \mathrm{~kg} \mathrm{~s}^{\mathrm{n-2}} / \mathrm{m}$ \\
\hline Reference temperature & $T_{\mathrm{o}}$ & $310^{\circ} \mathrm{K}$ \\
\hline
\end{tabular}

TABLE III: PARAMETERS OF CONTROL VOLUMES

\begin{tabular}{|l|c|}
\hline \multicolumn{1}{|c|}{ Mesh } & No. of control volume \\
\hline Finest & $1.44 \mathrm{M}$ \\
\hline Medium & $1.29 \mathrm{M}$ \\
\hline Coarse & $1.19 \mathrm{M}$ \\
\hline
\end{tabular}

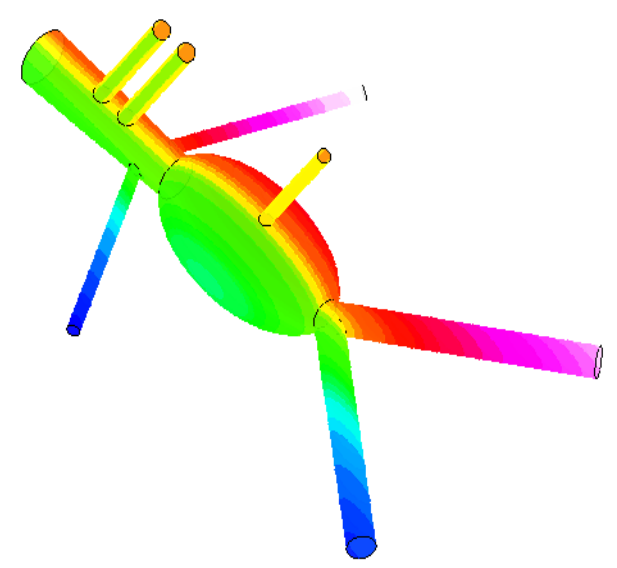

(a) 


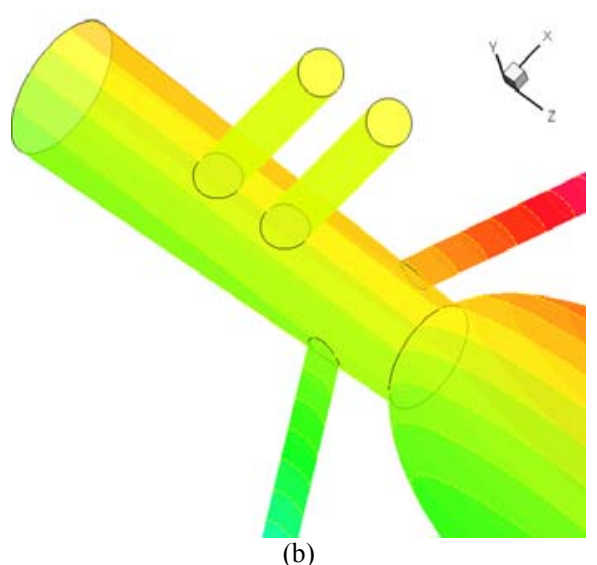

Fig. 2(a). Computational model of abdominal aortic aneurysm (b). Close up of peripheral branches

Therefore, behavior of blood should be taken into account while modeling the behavior of blood. In the present work, non-Newtonian Power law is applied for modeling blood; utilized parameters are shown in Table II. Non-Newtonian power law is described as:

$$
\begin{gathered}
\eta=k \dot{\gamma}^{n-1} e^{T o / T} \\
\eta_{\min }<\eta=k \dot{\gamma}^{n-1} e^{T o / T}<\eta_{\max }
\end{gathered}
$$

where $k$ is the average viscosity of the fluid, $n$ is the deviation of the fluid from Newtonian, To is the reference temperature, and $\eta_{\min } \& \eta_{\max }$ are the lower and upper limits of the non-Newtonian power law, used to predict the class of fluid.

\section{RESULTS}

CFD simulations were executed on three successively refined meshes (table III) to assessed grid independency.

All simulations were carried out at Computational Thermo-fluid Laboratory, NEDUET. Ten AMD Opteron Quad core CPUs of $2.4 \mathrm{GHz}$ each has been used,numerical analysis took 7-8 hours to converge, using Fluent v6.3.26.

\section{A. Flow dynamics}

Disturbance induced in the behavior of blood flow in AAA is predicted by modeling it for systolic as well as diastolic cardiac phases, separately. Fig. 4 revealed the flow dynamics in thecomputational domain. The generated profile in both phases indicates that the sudden large space developed due to aneurysm enforces flow to become highly turbulent. Convulsive flowinduces additional stressesand intensified concentrated pressure across the aneurysmal sac. It is believing that these are the possible roots of abnormal ballooning of aneurysm and enfeeblement of the aortic wall. Reverse flow at different sections and re-circulating vortices along the aneurismal sac is observed. Circulating vorticesincreased the likelihood of thrombosis and blood clot formation inside the blood vessel because of velocity drop. Fig. 4 reflects; there is considerably lesser frequency of reverse flow in systolic phase in comparison to diastolic phase but magnitude of reverse flow in systolic.

Recirculating vortices are present in both cardiac phases. However, diastolic phase is characterized with more vortices in comparison to the systolic, thus we can infer increased pressure reduces the frequency of vortex but increases the reverse flow jerk, its occupancy and magnitude. Figure 5 depicts the formation of vortex, in which it is clearly illustrated vortex tends to disappear in systolic cardiac phase. Fig. 4 and Fig. 5 correspond with [14] that the abnormal large space developed due to aneurysm in the AA consequences in a highly disturbed flow regime within AAA, which results in additional stresses across the aneurismal aortic wall accompanied by formation of re-circulating vortices, reverse flow and decreased blood velocity inside aneurysm and at the iliac bifurcation of AA, which is a favorable condition for the formation of blood clots. Blood clotting in AAA may result in the blockage of blood supply. Blood clot may break and form emboli because of a large pressure gradient. Emboli have a natural tendency of firmly attachment with blood vessels that results in the cut down of blood and oxygen supply to the concerned tissue that leads to a serious disorder (ischemia), which may results in tissue damage even tissue death of the concerned area[15].

Velocity profile and pressure distribution through the AA with AAA is shown in Fig. 6 and Fig. 7. Fig. 6 revealed velocity profile of blood flow through the abdominal aorta with aneurysm for both diastolic and systolic phases. Maximum velocity of $0.85 \mathrm{~m} / \mathrm{s}$ in case of diastolic phase whereas $0.96 \mathrm{~m} / \mathrm{s}$ in systolic phase, and a minimum velocity of $0 \mathrm{~m} / \mathrm{s}$ has been observed, which shows close resemblance with N.F Muhammed et al. [16] and Finol et al. [17]. Significant velocity drop is found in aneurysm $(0 \mathrm{~m} / \mathrm{s})$ paired with a reverse flow, which may give raises to the phenomenon of blood clotting within the aneurysm.

Maximum velocity $(0.85 \mathrm{~m} / \mathrm{s} \& 0.96 \mathrm{~m} / \mathrm{s})$ that is found at the renal bifurcation in both cardiac phases is due to theformation of a large pressure gradient between renal and iliac bifurcation (Fig. 7 (c) and Fig. (f))

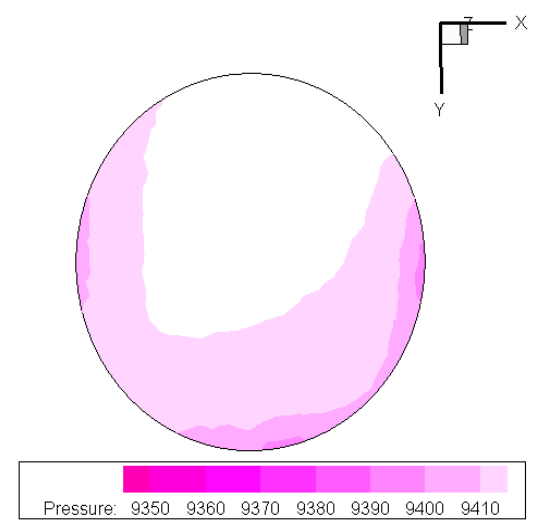

(a) Pressure contour at $z=0.08613$

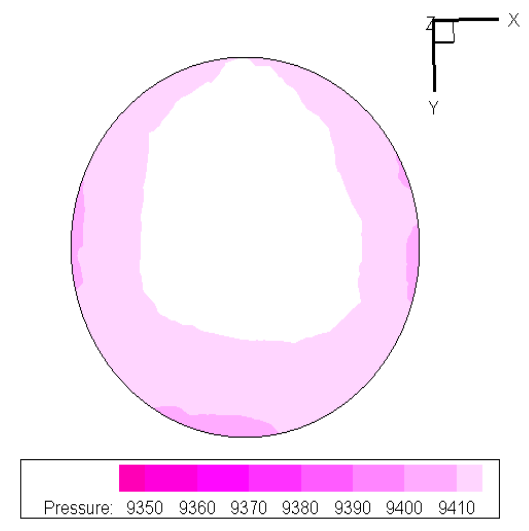

(b) Pressure contour at $z=0.08613$

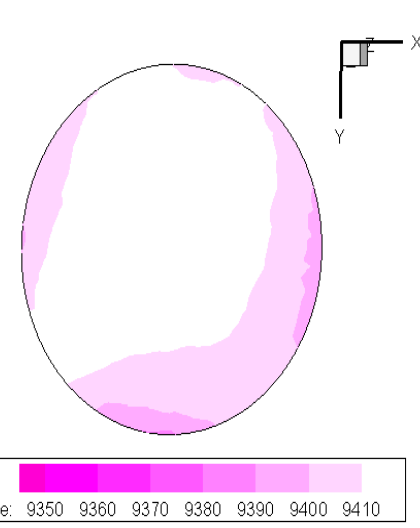

(c) Pressure contour at $z=0.08613$ 


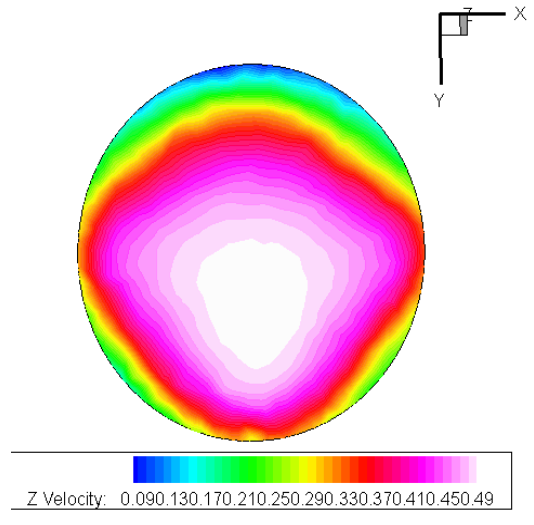

(d) Velocity contour at $z=0.08613$

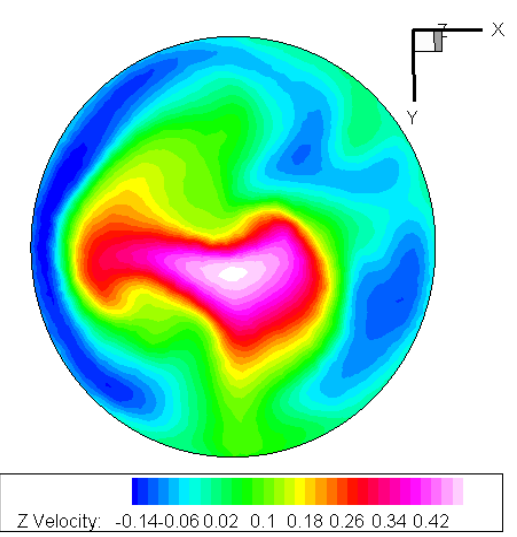

(g) Velocity contour at $z=14068$

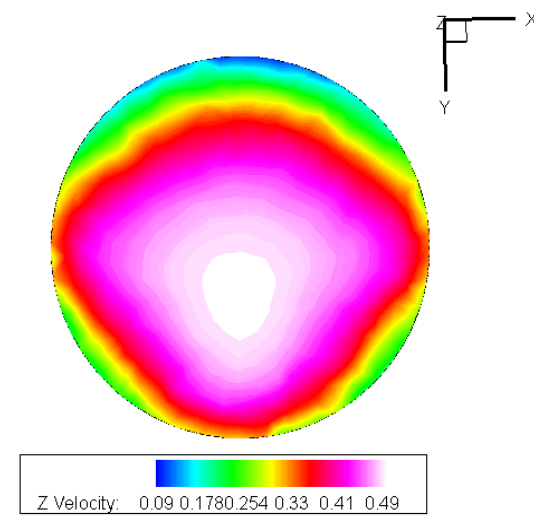

(e) Velocity contour at $z=0.08613$

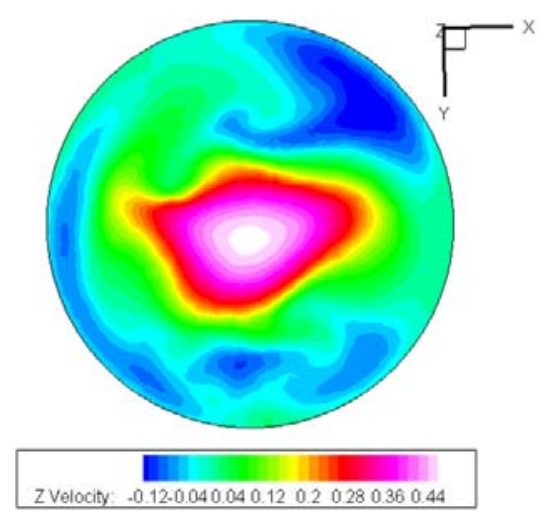

(h) Velocity contour at $z=14068$

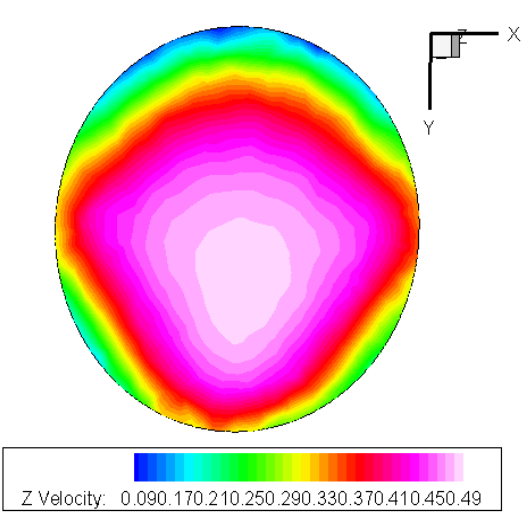

(f) Velocity contour at $z=0.08613$

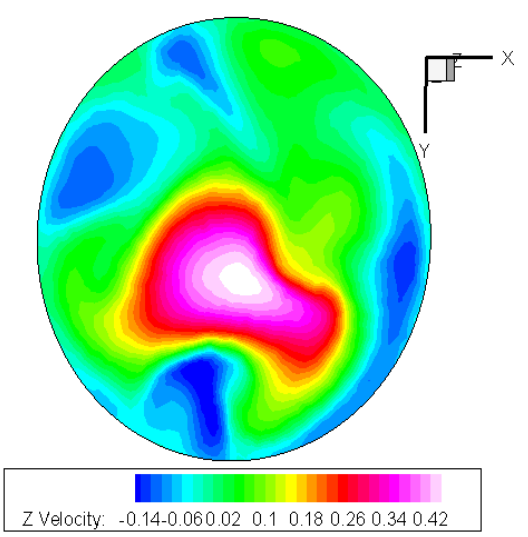

(i) Velocity contour at $z=14068$
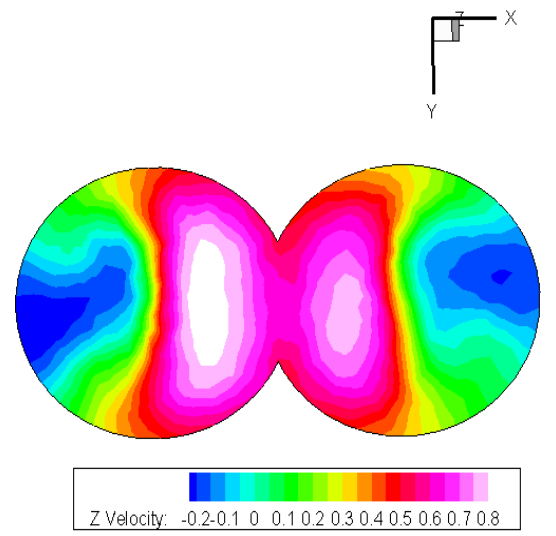

(j) Velocity contour at $z=0.19522$

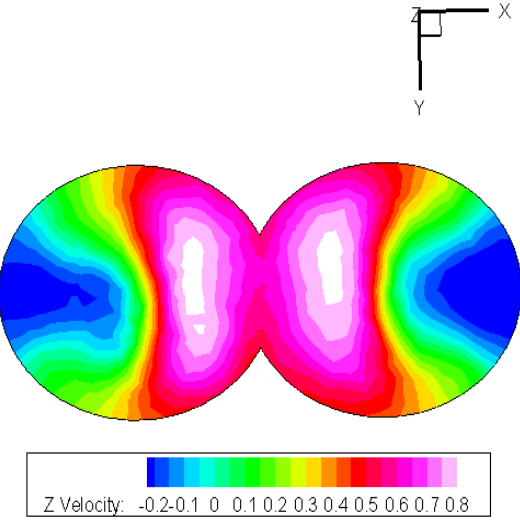

(k) Velocity contour at $z=0.19522$

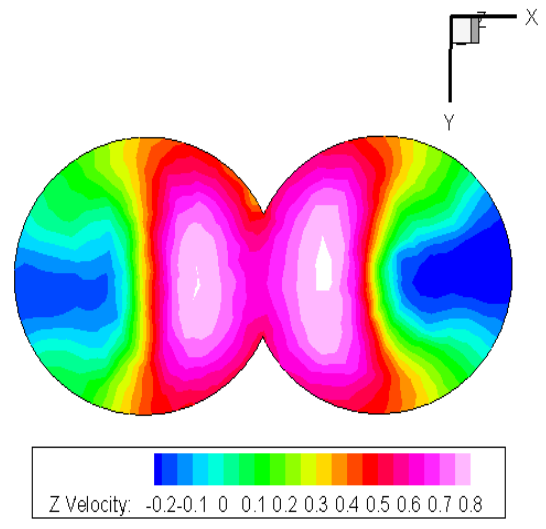

(1) Velocity contour at $z=0.19522$

Fig. 3. Depicts the flow profile through AAA in diastolic phases for all the three case

Profile for finest grid is shown in Fig (a), (d), (g), (j), For intermediate grid is shown in fig (b), (e), (h), (k), for coarse grid is shown in fig (c), (f), (i), (l)

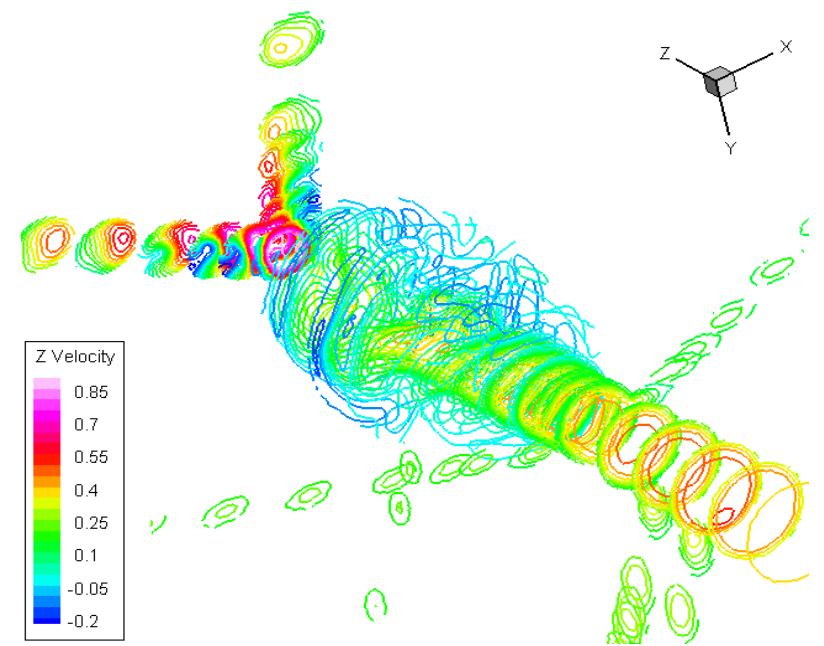

(a)

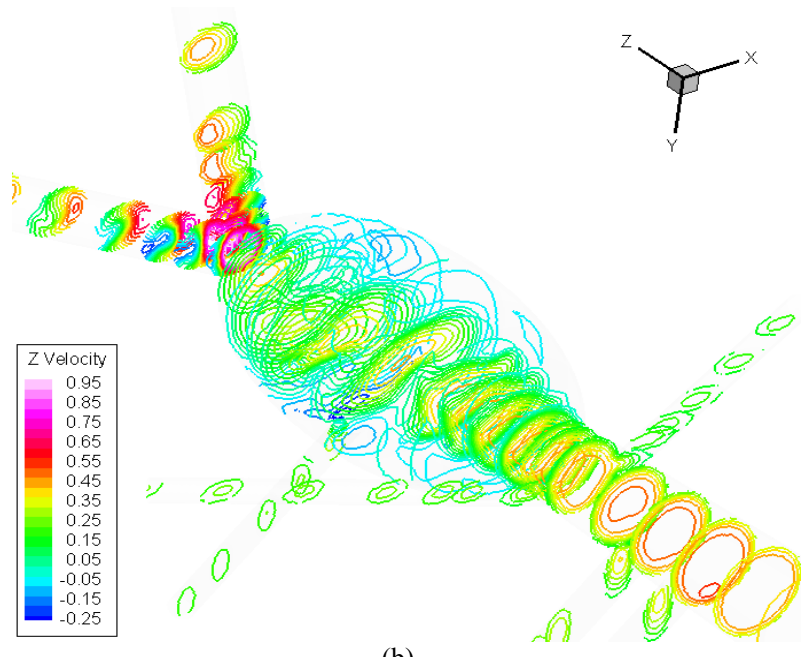

(b)

Fig. 4. (a) Streamlines for diastolic phase (b) Streamlines for systolic phase 


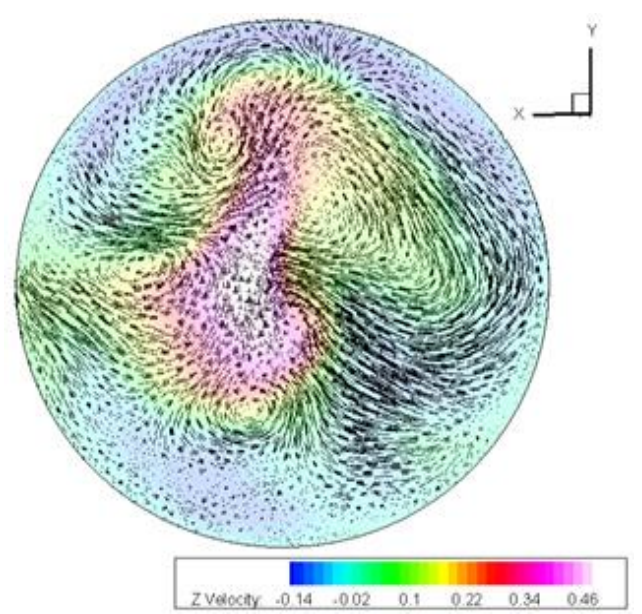

Fig. 5(a). Depicts vortices in aneurysmal sac for diastolic phase

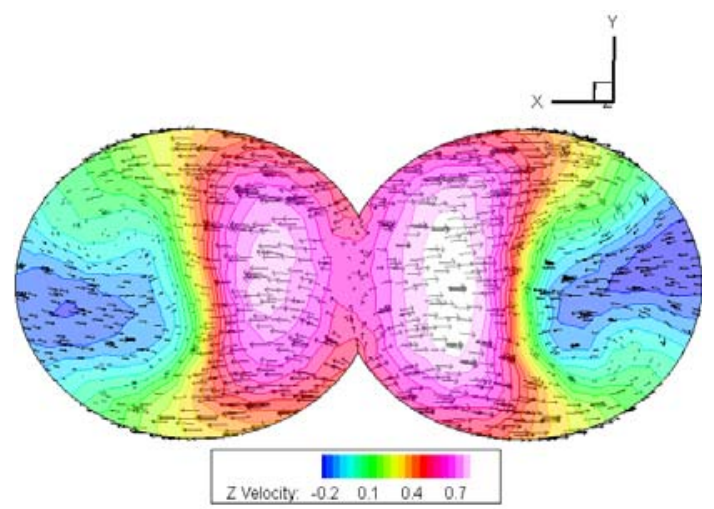

Fig. 5(c). Depicts reverse flow at iliac bifurcation for diastolic phase

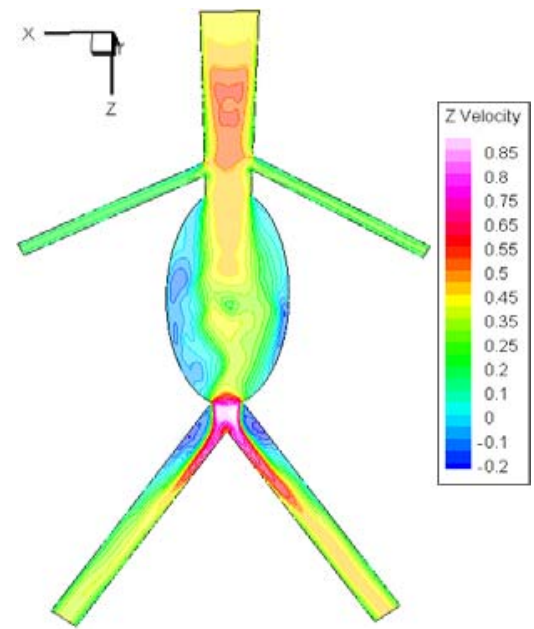

(a) Cross-sectional slice taken at $z=0$ for diastolic phase

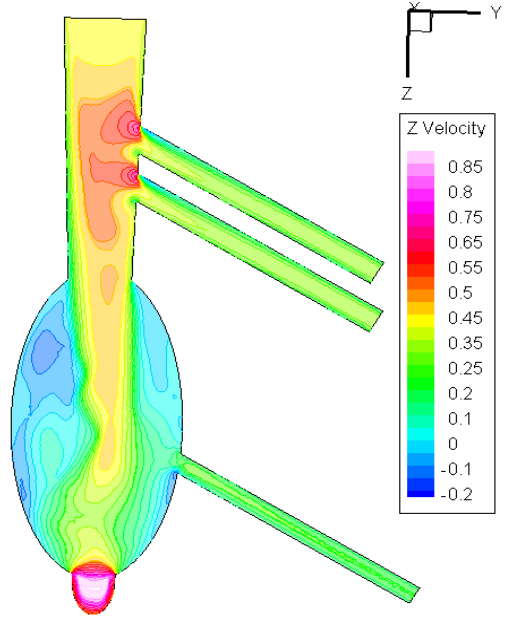

(c) Cross-sectional slice taken at $x=0$ for diastolic phase

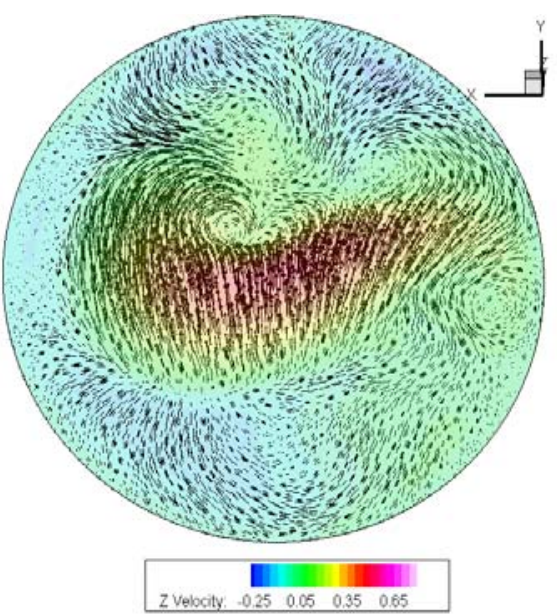

Fig. 5(b). Depicts vortices in aneurysmal sac for systolic phase

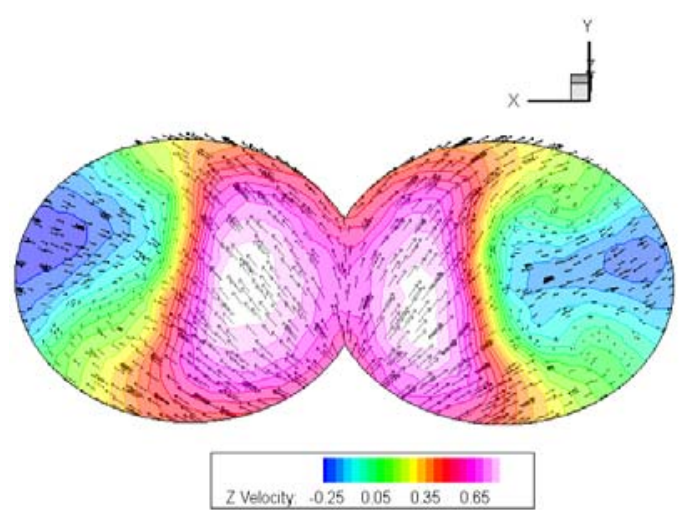

Fig. 5(d). Depicts reverse flow at iliac bifurcation for systolic phase

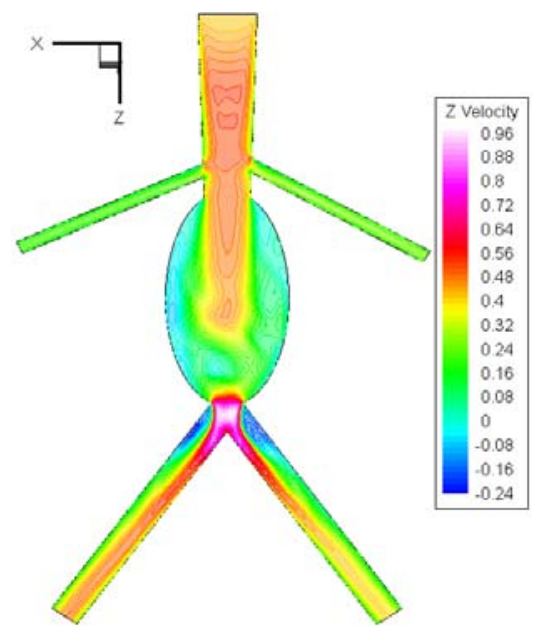

(b) Cross-sectional slice taken at $z=0$ for diastolic phase

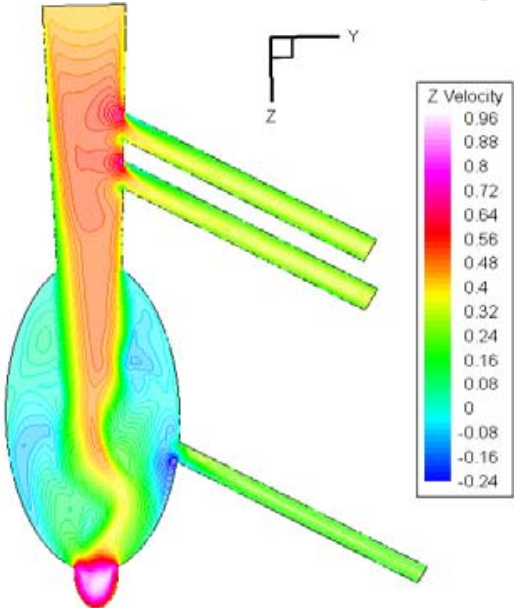

(d) Cross-sectional slice taken at $x=0$ for systolic phase Fig. 6. Depicts velocity profile through cross-sectional slice of AA with aneurysm for systolic and diastolic phases 


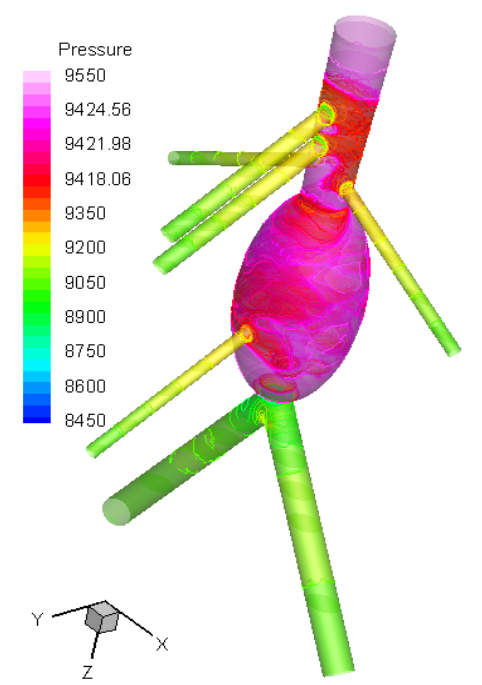

(a) Overall pressure distributions in diastolic Phase

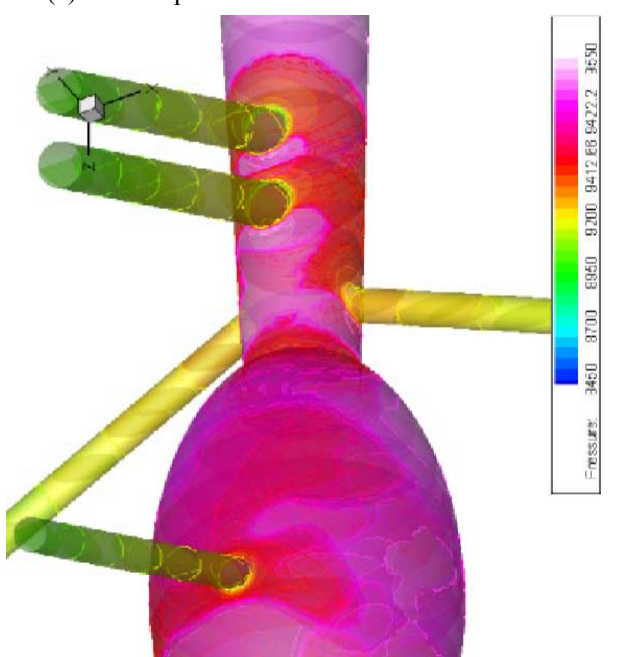

(c) Close up of peripheral branches in diastolic phase

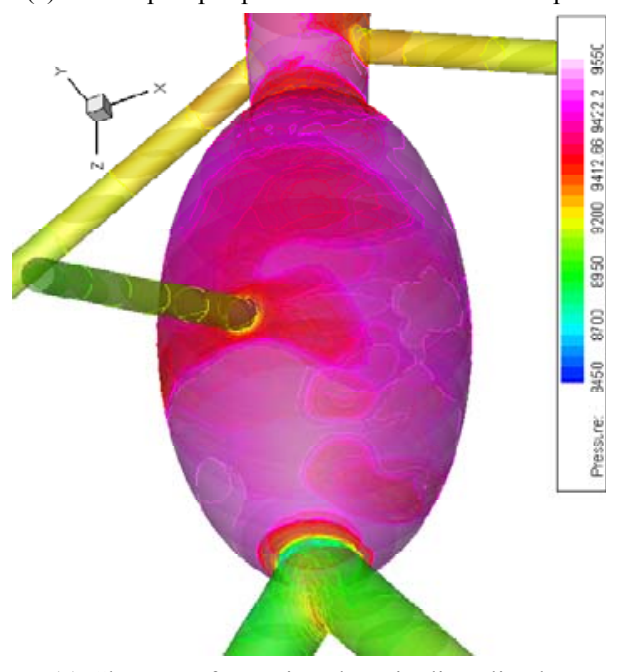

(e) Close up of aneurismal sac in diastolic phase

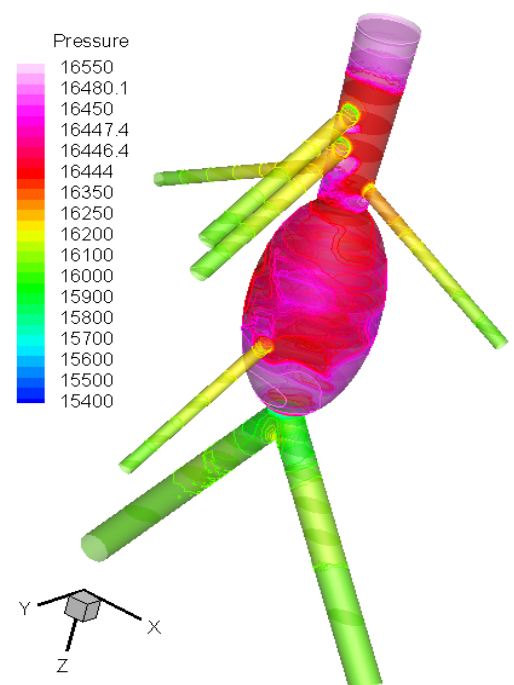

(b) Overall pressure distributions in diastolic phase

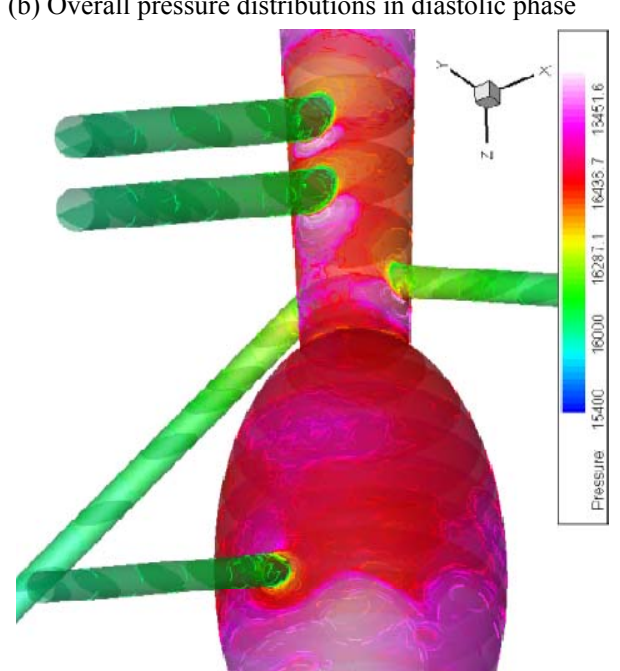

(d) Close up of peripheral branches in systolic phase

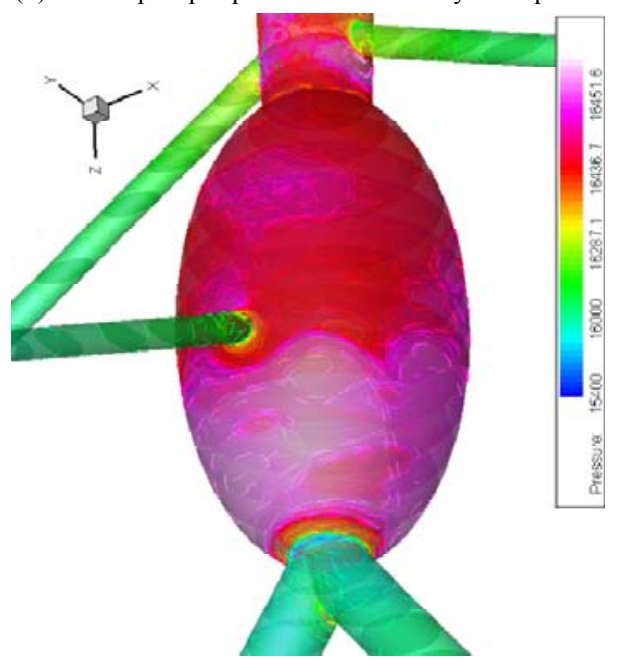

(f) Close up of aneurismal sac in systolic phase

Fig. 7. Depicts pressure distribution through Abdominal Aorta with aneurysm

Fig. 7 revealed the pressure distribution through the abdominal aorta with aneurysm. Aneurismal sac is characterized with higher pressure in comparison to remaining. It is found that the pressure distribution is temporally diverging throughout the domain of AA with AAA ranging from $8450 \mathrm{~Pa}-9550 \mathrm{~Pa}$ (in diastolic phase) and $15400 \mathrm{~Pa}-16550 \mathrm{~Pa}$ (in systolic phase) which exhibits excellent agreement with Leung et al. [18], and Hassaniet al. [19]. The pressure distribution through the AAA shows that the highest pressure (9412 $\mathrm{Pa}, 16436 \mathrm{~Pa}$ ) is found at the inlet of aneurysm and minimum pressure $(8920 \mathrm{~Pa}, 16000)$ at the outlet which builds up a huge pressure gradient of (492 Pa, $436 \mathrm{~Pa}$ ) in both respective cardiac phases. Peak pressure is found to be at same location in both cardiac phases, i.e. it is not a function of cardiac phases. However, because of the large space and pressure gradient a continued disturbance in velocity profile is observed throughout AA. 


\section{CONCLUSIONS}

In this paper, we have analyzed the behavior blood flow through AA with aneurysm by employing FVM. It validates pressure buildup between the iliac \& renal bifurcation is the responsible for uninterrupted ballooning of AAA. It is observed abrupt expansion of AA results in highly turbulent flow within the AAA. Therefore, we arelooking forward to studying hemodynamic indicators and wall shear stress by turbulence modeling in coming months along with the study of viscosity effect on aneurismal sac.

\section{ACKNOWLEDGMENT}

We would like to thank the staff of Computational Thermo-fluid Laboratory \& Higher Performance of Computing centre, NED University of Engineering \& Technology, for their support throughout this research work.

\section{REFERENCES}

[1] Henry Gray, "VI. The Arteries. 5a. 2. The Abdominal Aorta. Gray, Henry. 1918. Anatomy of the Human Body," [Online]. Available: http://www.bartleby.com/107/154.html [Accessed:Jul.25,2010].

[2] Anonymous, "AbdominalAorticAneurysm," [Online]. Available: http://www.vascularweb.org/vascularhealth/Pages/AbdominalAorticA neurysm.aspx [Accessed:Jul.25,2010].

[3] David A. Vorp, David A. Steinman, C. Ross Ethier, "Computational modeling of arterial biomechanics", Computing in Science and Engineering, vol. 3, pp. 51, 2001.

[4] Mark W. Siebert and Petru S. Fodor, "Newtonian and non-newtonian blood flow over a backward-facing step - a case study", proceeding of the COMSOL Conference Boston 2009.

[5] D. Lee, J. Y. Chen, "Pulsatile flow fields in a model of abdominal aorta with its peripheralbranches", Biomedical Engineering: Applications, Basis and Communications, vol.15, pp.170, October 2003.

[6] D. Lee, J.Y. Chen, "Numerical simulation of steady flow fields in a model of abdominalaorta with its peripheral branches", Journal of Biomechanics, vol. 35, pp. 1115, August 2002.

[7] Charles A. Taylor, Thomas J. R. Hughes, And Christopher K. Zarins, "Finite element modeling of three-dimensional pulsatile flowin the abdominal aorta: relevance to atherosclerosis", Annals of Biomedical Engineering, vol. 26, pp. 975, November 1998.

[8] Ender A. Finol, Cristina H. Amon, "Blood flow in abdominal aorticaneurysms: pulsatile flowhemodynamics", Journal of Biomechanical Engineering, vol. 123, pp. 474, October 2001.

[9] Kamran Hassani, Mahdi Navidbakhshand Mostafa Rostami, "Modeling of the aorta artery aneurysms and renal artery stenosisusing cardiovascular electronic system" BioMedical Engineering OnLine, vol. 6, pp. 22, June 2007.

[10] Michael P. Poullis, Richard Warwick, AungOo and Robert J. Poole, "Ascending aortic curvature as an independent risk factor for type a dissection, and ascending aortic aneurysm formation:a mathematical model", European Journal of Cardio-Thoracic surgery, vol. 33, pp. 995-1001, June 2008.

[11] Khanafer KM, Bull JL, Upchurch GR Jr, Berguer R., "Turbulence significantly increases pressureand fluid shear stress in an aortic aneurysmmodel under resting and exercise flowconditions", Annals of Vascular Surgery, vol. 21, pp. 67, January 2007.
[12] Fung, Y. C., "The Flow Properties of Blood", in Biomechanics Mechanical Properties of Living Tissues, Springer, 2004, ch. 3, pp. 62-99.

[13] C. M. Rodkiewicz, PrawalSinha, J.S. Kennedy, "On the application of a constitutive equation for whole human blood", vol. 112, pp. 198. May 1990.

[14] Sandor IBernad, Elena S. Bernad, TiberiuBarbat, Romeo SusanResiga, VladAlbulescu, "Effects of asymmetry in patient-specific wall shear stess analyses ofabdominal aortic aneurysm", Journal of Chinese Clinical Medicine, vol. 4, pp. 424, 2009.

[15] Updated by: Linda J. Vorvick, Yi-Bin Chen, David Zieve, "Blood clots: MedlinePlus Medical Encyclopedia," [Online].Available: http://www.nlm.nih.gov/medlineplus/ency/article/001124.htm [Accessed:Jul.25,2010]

[16] N.F Mohammad, M. N. Nassriq, A. Saidatul "Analysis of blood flow, pressure and velocity in a stented abdominal aortic aneurysm model" Proceedings of International Conference on Applications and Design in Mechanical Engineering (iCADME), October 2009.

[17] Ender A. Finol and Cristina H. Amon "Flow dynamics in anatomical models of abdominal aortic aneurysms: computational analysis of pulsatile flow" ActaCientíficaVenezolana, vol. 54, pp. 43, 2003.

[18] Kamran Hassani, Mahdi Navidbakhsh and Mostafa.Rostami, "Simulation of aorta artery aneurysms using active electronic circuit" American Journal of Applied Sciences, vol. 4, pp. 2003, 2007.

[19] James H Leung, Andrew R Wright, Nick Cheshire Jeremy Crane, Simon A Thom, Alun D Hughes and Yun $\mathrm{Xu}$, "Fluid structure interaction of patient specific abdominal aortic aneurysms: a comparison with solid stress models" BioMedical Engineering OnLine, vol. 5, pp. 33, 2006

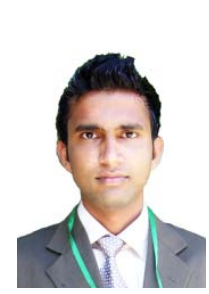

Muhammad Amir Azam received his B.E in Biomedical Engineering from NED University of Engineering and Technology. His research interests are in the area of Programming, Robotics, Modeling and Simulation and fluid dynamics. His research on these and other topics has appeared in international conferences like Euromech Fluid Mechanics Conference (EFMC), International Conference on Machine Learning and Computing (ICMLC), etc. His book titled "Mobile Robots Paradigm Approach for Designing \& Development of Algorithms for Autonomous Path Planning and Navigation (ISBN 978-3-8443-3384-8)" has been published by LAMBERT Academic publishers (Germany). When he is not busy in teaching and writing, he enjoys reading all different types of books, and many different activities like playing Chess with friends.

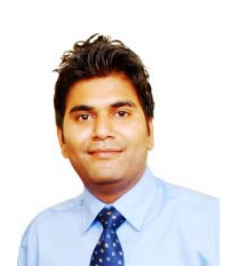

Syed Abu Anzar Salam is a visiting lecturer in Dow University of Health \& Sciences\& Indus Institute of Higher Education. He did his graduation in Medical Engineering from NED University of Engineering \& Technology. $\mathrm{He}$ is always associated with key educational institutes of Pakistan like NEDUET, DUHS, \&Adamjee Govt. Science College. He is recognized for innovation, research, managerial skills, $\&$ ability to do. He is keenly interested in Biofluid, Biomechanics, Robotics \& Control, and Computational Modeling \& simulations. He enjoyed multiple paper publication in international conferences like EFMC, ICMLC, etc. His book titled "Mobile Robots Paradigm Approach for Designing \& Development of Algorithms for Autonomous Path Planning and Navigation (ISBN 978-3-8443-3384-8)" has been published by LAMBERT Academic publishers (Germany). He has diversified experience of $R \& D$ and teaching ranging from schooling to undergraduate students. 